\title{
ERRATA
}

\section{Observation of the Local Structure of Landau Bands in a Disordered Conductor [Phys. Rev. Lett. 78, 1540 (1997)]}

T. Schmidt, R. J. Haug, Vladimir I. Fal'ko, K. v. Klitzing, A. Förster, and H. Lüth

[S0031-9007(97)03212-2]

Figure 3 was intended for color publication but was not printed in color. Because of this error, we reprint the entire Letter here. 


\title{
Observation of the Local Structure of Landau Bands in a Disordered Conductor
}

\author{
T. Schmidt, ${ }^{1}$ R. J. Haug, ${ }^{1,3}$ Vladimir I. Fal'ko, ${ }^{1,4}$ K. v. Klitzing, ${ }^{1}$ A. Förster, ${ }^{2}$ and H. Lüth ${ }^{2}$ \\ ${ }^{1}$ Max-Planck-Institut für Festkörperforschung, Heisenbergstrasse 1, 70569 Stuttgart, Germany \\ ${ }^{2}$ Institut für Schicht- und Ionentechnik, Forschungszentrum Jülich GmbH, Postfach 1913, 52428 Jülich, Germany \\ ${ }^{3}$ Institut für Festkörperphysik, Universität Hannover, Appelstrasse 2, 30167 Hannover, Germany \\ ${ }^{4}$ School of Physics \& Chemistry, Lancaster University, LA1 4YB Lancaster, United Kingdom
}

(Received 31 October 1996)

\begin{abstract}
The local density of states of heavily doped GaAs is explored at high magnetic fields, where only a single or few Landau bands are occupied. Our experiment is based on resonant tunneling through impurity states and images the local density of states both below and above the Fermi level. Fantype mesoscopic fluctuations are observed in the energy-magnetic-field plane, which we attribute to the interplay of Landau quantization and quantum interference of scattered electron waves in the disordered conductor. Our conclusion is supported by the suppression of the fluctuations high above the Fermi level, where dephasing due to inelastic processes is as fast as elastic scattering. [S0031-9007(97)02403-4]
\end{abstract}

PACS numbers: 73.23.Hk, 73.23.Ps, 71.23.-k, 71.70.Di

Landau quantization leads to singularities in the density of states (DOS) of three-dimensional (3D) conductors. Therefore, many physical quantities exhibit an oscillatory dependence on magnetic field, a prominent example being the Shubnikov-de Haas oscillations of the conductance. At low magnetic fields, impurity scattering dominates cyclotron motion in disordered metals, and both the transport properties and the DOS show mesoscopic fluctuations due to quantum interference of diffusive electron waves [1]. However, little is known about mesoscopic effects in the regime of high magnetic fields, except that cyclotron motion and Landau quantization strongly modify quantum diffusion $[2,3]$.

The local density of states (LDOS) is particularly sensitive to disorder [1,4-6]. Recently, we developed a technique to image the LDOS in heavily doped GaAs [7], which is based on resonant tunneling through impurity states $[5,8-10]$ in asymmetric double-barrier heterostructures. In agreement with theory [4,5], we found at low magnetic fields strong irregular LDOS fluctuations [7]. Interesting mesoscopic structure was also observed at high fields [11], which suggests that the LDOS allows to explore the interplay of cyclotron motion and quantum diffusion in disordered conductors.

In this Letter, we investigate the LDOS of heavily doped $\mathrm{GaAs}$ in the regime of quantizing magnetic fields, where only a single or few Landau bands are occupied. The LDOS is found to exhibit pronounced fan-type resonances in the energy-magnetic-field plane, which disappear high above the Fermi level. We relate these LDOS resonances to the formation of quasi-1D singleparticle states in the disordered 3D system due to the influence of Landau quantization on the propagation of scattered electron waves.

Our experiment is based on a strongly asymmetric double-barrier heterostructure, which was grown by molecular-beam epitaxy on an $n^{+}$-type GaAs substrate. It consists of a $10 \mathrm{~nm}$ wide GaAs quantum well sandwiched between two $\mathrm{Al}_{0.3} \mathrm{Ga}_{0.7} \mathrm{As}$ barriers of 5 and $8 \mathrm{~nm}$ width, respectively (top and bottom barrier). The nominally undoped active region is separated from $300 \mathrm{~nm}$ thick GaAs contact layers doped with Si to $4 \times 10^{17} \mathrm{~cm}^{-3}$ by $7 \mathrm{~nm}$ narrow GaAs spacer layers. The doping corresponds to a Fermi energy of $30 \mathrm{meV}$. From this material we fabricated a $1 \mu \mathrm{m}$ diameter mesa with Ohmic contacts. Most probably due to growth-induced donor segregation, the sample contains a few impurities in the quantum well.

The operation of our device is illustrated in Fig. 1. We use the energetically lowest impurity state $S$ as spectrometer for the LDOS in the left contact adjacent to the thick barrier. By applying a bias voltage $V$, the spectrometer is shifted in energy with respect to both contacts. For $V>0$, the left contact acts as electron emitter (and the right one as collector) [Fig. 1(a)]. In contrast, for $V<0$ the left contact is the collector (and the right one the emitter) [Fig. 1(b)]. Independent of the

(a)

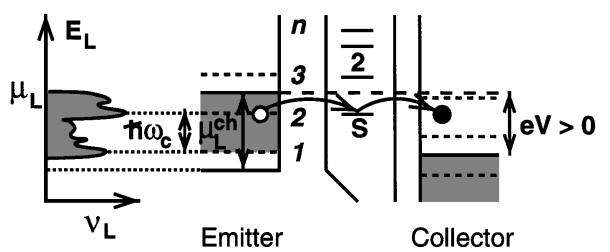

(b)

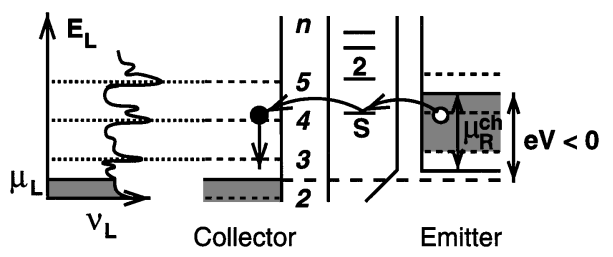

FIG. 1. Conduction-band profile of the strongly asymmetric double-barrier heterostructure for (a) $V>0$ and (b) $V<0$. Independent of the bias polarity, the LDOS in the left contact adjacent to the thick barrier is under investigation. 
bias polarity, $S$ crosses the Fermi level in the emitter at

$$
\left|V_{S}\right|=\left(E_{S}-\mu_{L, R}^{c h}\right) / e \alpha
$$

and a current step results from resonant tunneling. Here, $E_{S}$ is the energy of $S, \mu_{L}^{c h}=\mu_{R}^{c h}$ are the chemical potentials in the contacts (measured from the band edge in the well and the contacts, respectively), and $\alpha$ is the voltage-to-energy conversion coefficient. The tunneling current is basically determined by the tunneling rate of the thick left barrier which is much lower than that of the right one, i.e., $\Gamma_{L} \ll \Gamma_{R}$. For $V>$ 0 , the current is given by $I=2 e \Gamma_{L} \Gamma_{R} /\left(2 \Gamma_{L}+\Gamma_{R}\right) \approx$ $2 e \Gamma_{L}$, whereas $I=2 e \Gamma_{L} \Gamma_{R} /\left(\Gamma_{L}+2 \Gamma_{R}\right) \approx e \Gamma_{L}$ results for $V<0$. The difference stems from Coulomb blockade which prevents double occupancy of $S[12,13]$. As $\Gamma_{L}$ is proportional to the local tunneling density of states $\nu_{L}$ in the left contact at the position of $S$, the current $I \propto \nu_{L}$ images for $V>0$ the LDOS of occupied states below the Fermi level $\mu_{L}$ and for $V<0$ the LDOS of empty states above $\mu_{L}$. Hence, the differential conductance $G=d I / d V \propto d \nu_{L} / d E$ displays the derivative of the LDOS with respect to energy. At a bias voltage of

$$
V_{L}=\left(E_{S}-E_{L}\right) / e \alpha
$$

the LDOS at energy $E_{L}$ is probed by our experiment (measured from the band edge in the left contact).

Both the $I(V)$ and $G(V)$ characteristics of the device were measured independently in a dilution refrigerator. Figure 2(a) shows the low-bias region of the $I(V)$ curve recorded at the base temperature of $T=30 \mathrm{mK}$ in a magnetic field of $B=12 \mathrm{~T}$ directed parallel to the current flow. Two current steps are clearly observed for both bias polarities due to resonant tunneling through the two lowest discrete levels $S$ and 2 (Fig. 1). The first step occurs almost symmetrical with respect to zero bias, i.e., $\alpha \approx 0.5$ is independent of the bias polarity; see Eq. (1) [14]. For $V>0$, the data exhibit an oscillatory fine structure on the current plateau between the steps, which reflects LDOS fluctuations of occupied states in the left

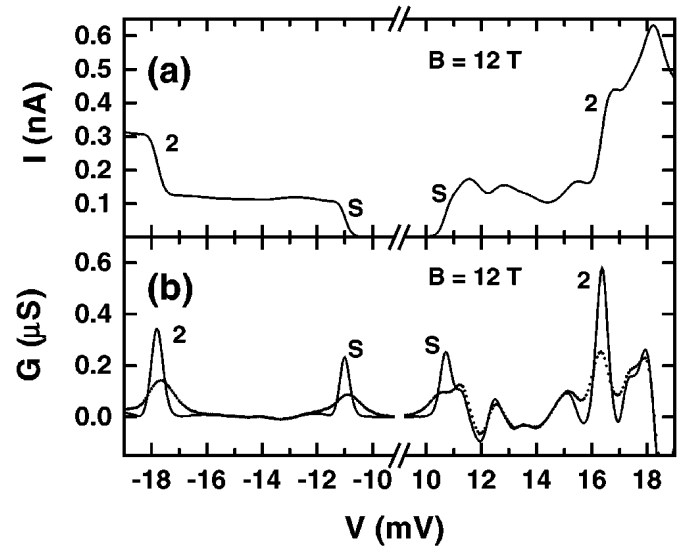

FIG. 2. (a) Current-voltage curve $I(V)$ measured at $T=$ $30 \mathrm{mK}$. (b) Differential conductance $G=d I / d V$ recorded at $T=30 \mathrm{mK}$ (solid line) as well as $T=1.8 \mathrm{~K}$ (dotted line). contact [7]. Indications of fine structure are also visible for $V<0$, particularly in the differential conductance plotted in Fig. 2(b) for $T=30 \mathrm{mK}$ and $1.8 \mathrm{~K}$. Large peaks originate in the $G(V)$ curves from the steps in the $I(V)$ data. The fine structure between these peaks is independent of temperature, since it results from resonant tunneling from the emitter below the Fermi level (where all states are occupied) through $S$ into the collector above the Fermi level (where all states are empty).

Now, we address the influence of high magnetic fields on the LDOS in disordered 3D conductors. Landau quantization is relevant, if the cyclotron frequency $\omega_{c}=$ $e B / m^{*}$ exceeds the electron scattering rate $\tau^{-1}=v_{F} / l$ (with $v_{F}$ the Fermi velocity of the electrons and $l$ their elastic mean free path). For the contacts of our device, we estimate $\omega_{c} \tau>1$ to be satisfied above $B=$ 1.5-3 $\mathrm{T}$ (deducing $l=50-100 \mathrm{~nm}$ from the doping density). In case of negligible disorder, electron states in magnetic field obey $E_{L}=(n+1 / 2) \hbar \omega_{c}+E\left(k_{z}\right)$, with $n=0,1, \ldots$ and $E\left(k_{z}\right)$ the kinetic energy of fieldparallel free motion. At low fields, disorder leads to strong LDOS fluctuations due to quantum interference of diffusive electron waves [1,4,7]. At high fields, the electron states of disordered systems assume a quasi-1D character, because diffusion perpendicular to the magnetic field is suppressed by the Lorentz force [2,3]. Sets of LDOS resonances $\beta$ with longitudinal energies $E^{\beta}=$ $E\left(k_{z}\right)$ are formed by quasi-1D interference of multiplyscattered electron waves subject to Landau quantization. The magnetic-field dependence of the resonance energies

$$
E_{L}=(n+1 / 2) \hbar \omega_{c}+E^{\beta}
$$

is linear, until individual resonances are destroyed due to the modification of the scattering conditions by the changing magnetic length. From Eqs. (2) and (3), we infer that the LDOS resonances in the left contact generate a fan-type pattern of negative slope $d V_{L} / d B<0$ in the bias-voltage-magnetic-field plane.

Figure 3 shows a color-scale image of $G=d I / d V$ as a function of bias voltage and magnetic field, which is dominated by black lines of large differential conductance. The two lowest lines denoted by $V_{S}$ and $V_{2}$ correspond to the peaks of the $G(V)$ curves in Fig. 2. Note that the color scale is for $V<0$ more sensitive than for $V>0$. At $B>2 \mathrm{~T}$, the fine structure between $V_{S}$ and $V_{2}$ exhibits indeed pronounced fan-type resonances for both bias polarities, as predicted above for the local structure of the Landau bands. In contrast, the fluctuations are strongly irregular at $B \leq 2 \mathrm{~T}$, where Landau quantization is irrelevant [7]. For $V>0$, the fan-type resonances move with increasing field to lower bias, $d V_{L} / d B<0$, as expected for LDOS fluctuations of occupied states in the left contact. In contrast, we observe two sets of resonances with different orientation for $V<0$. However, the dominant set shows again a negative slope, $d V_{L} / d B<0$, which demonstrates that it 


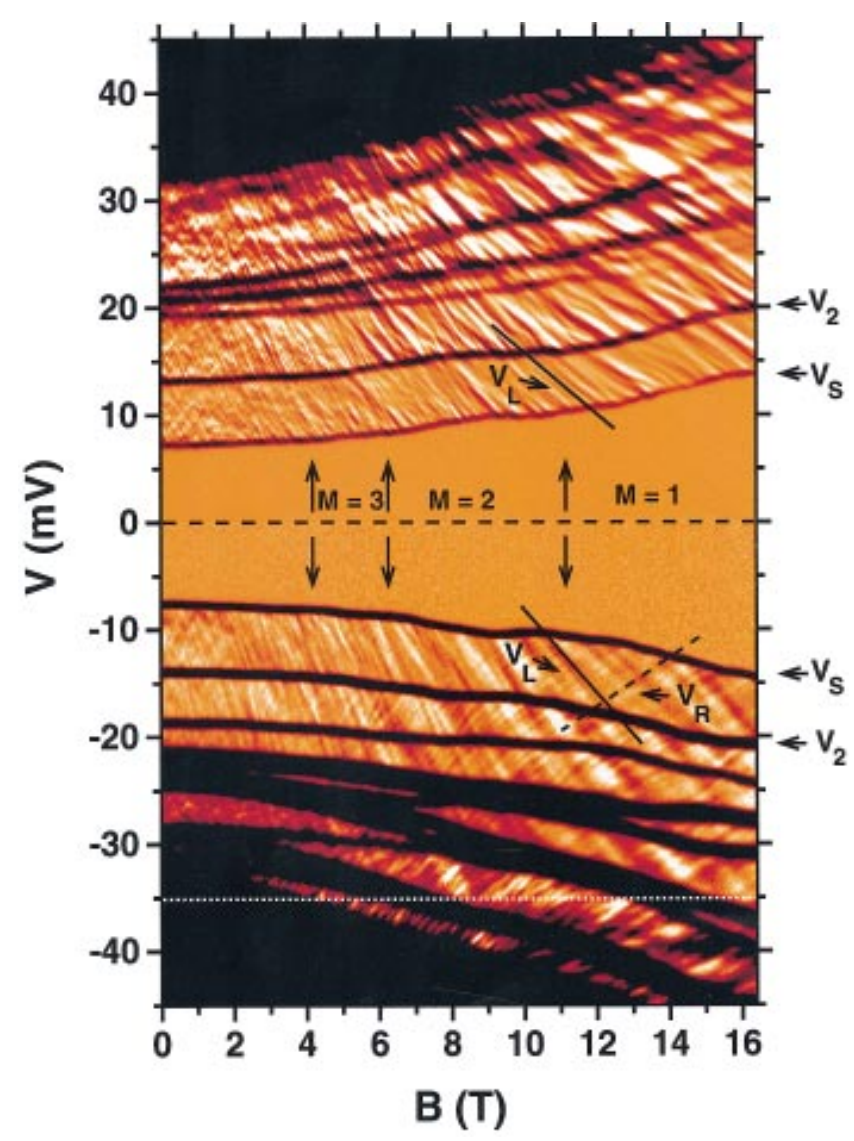

FIG. 3(color). Color map of the differential conductance vs magnetic field $B \| I$ (step $0.05 \mathrm{~T}$ ) and bias voltage (step $0.05 \mathrm{mV})$ for $T=30 \mathrm{mK}(V>0$ : white, $G \leq-0.2 \mu \mathrm{S}$; black, $G \geq 0.5 \mu \mathrm{S}$. $V<0$ : white, $G \leq-0.03 \mu \mathrm{S}$; black, $G \geq 0.07 \mu \mathrm{S})$.

reflects the LDOS of empty states in the left contact. The rudimentary set of positive slope $d V_{R} / d B>0$ arises from the right contact. Its orientation is different because the bias voltage moves $S$ energetically into opposite directions with respect to the two contacts (Fig. 1). By following individual LDOS resonances from $V_{S}$ through $V_{2}$, we find that some of them cross $V_{2}$, whereas others vanish. We attribute this result to the fact that beyond $V_{2}$ (and below the next black line), a superposition of LDOS fluctuations from two different points in space is reflected in our data (determined by the random positions of $S$ and 2 in the quantum well). For a different sample, we measured the fluctuation pattern not only for $B \| I$ but also for tilt angles of $\pi / 4$ and $\pi / 2$ (not shown). Fan-type resonances occur for all magnetic-field orientations, which complies with their bulk origin [15].

Before we further discuss the fan-type fine structure, we briefly focus on the spectrometer. With increasing magnetic field, $\left|V_{S}\right|$ moves to higher bias due to a diamagnetic shift of $E_{S}$ to higher energies [Eq. (1)]. To estimate the size of the spectrometer, we model its energy by $E_{S}=\sqrt{\left(\hbar \omega_{0}\right)^{2}+\left(\hbar \omega_{c} / 2\right)^{2}}$ and obtain $\hbar \omega_{0} \approx 29 \mathrm{meV}$ (using $\left|V_{S}\right| \approx E_{S} / e \alpha+$ const). For the radius of $S$, we evaluate $r \sim \sqrt{2 \hbar / m^{*} \omega_{0}} \sim 10 \mathrm{~nm}$ (cf. Ref. [16]), which corroborates that $S$ is a single donor in the quantum well. In addition, $\left|V_{S}\right|$ shows a series of dips which are fairly periodic in $1 / B$ (marked by arrows in Fig. 3). Such dips occur according to Eq. (1), when the chemical potential in the emitter contact reaches a maximum in energy, i.e., whenever a Landau band is depopulated [16]. Between consecutive dips, the number $M$ of partially occupied spin-degenerate Landau bands remains constant. All dips occur for both bias polarities at similar positions, since the left contact (emitter for $V>0$ ) and the right contact (emitter for $V<0$ ) have identical electron densities. The last dip at $B \sim 11.4 \mathrm{~T}$ reflects the transition to the magnetic quantum limit in which only one spindegenerate Landau band remains populated. From its position, we estimate the electron density in the contacts to $\left(\sqrt{2} / \pi^{2}\right)(e B / \hbar)^{3 / 2}=3.3 \times 10^{17} \mathrm{~cm}^{-3}$, which compares well with the donor concentration.

Now, we explore the fine structure in the regions between $V_{S}$ and $V_{2}$, which results exclusively from resonant tunneling through $S$. The corresponding energy interval, in which occupied states in the left contact are imaged for $V>0$, is directly located below $\mu_{L}$. Its width of $e \alpha \times 5 \mathrm{mV} \approx 2.5 \mathrm{meV}$ is narrow compared to the Fermi energy of $30 \mathrm{meV}$. The interval of energies, in which empty states are probed for $V<0$, has a similar width but starts at an energy of $e\left|V_{S}\right| \sim 10 \mathrm{meV}$ above $\mu_{L}$. As the LDOS is proportional to the current, we plot in Fig. 4(a) the average plateau current $\langle I\rangle_{V}$ of the first step vs magnetic field, where $\langle\ldots\rangle_{V}$ symbolizes averaging over bias voltage. For $V>0$, this quantity reflects an energy average over the LDOS of occupied states close to $\mu_{L}$. Minima slightly beyond the depopulation fields (marked by arrows) are reminiscent of the abrupt decrease of the global DOS at
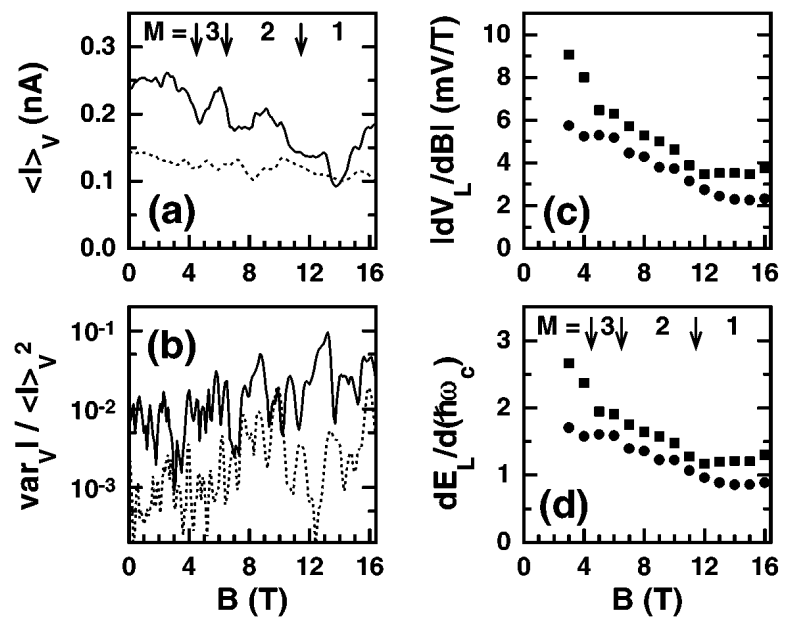

FIG. 4. (a) Average plateau current and (b) normalized variance of the current vs magnetic field. Solid lines correspond to $V>0$ and dotted lines to $V<0$. Slope of the (c) linear fine structure and (d) LDOS resonance energies vs magnetic field. Circles correspond to occupied states and squares to empty states in the left contact. 
the Fermi level when a Landau band is depopulated. For $V<0$, no pronounced structure is observed, probably because the LDOS's of both contacts contribute. Figure 4(b) shows the normalized variance $\operatorname{var}_{V} I /\langle I\rangle_{V}^{2}$ of the plateau current vs magnetic field, with $\operatorname{var}_{V} I=\left\langle\delta I^{2}(V)\right\rangle_{V}$ and $\delta I(V)=I(V)-\langle I\rangle_{V}$. The fluctuations grow strongly with increasing field, thus signifying the suppression of diffusion in the magnetic quantum regime [2,3].

Returning to the slope of the LDOS resonances, we show $\left|d V_{L} / d B\right|$ in Fig. 4(c). For both occupied and empty states, the slope decreases with increasing magnetic field, together with the number of available Landau bands. Moreover, the slope of empty states exceeds that of occupied states, because more Landau bands contribute at higher energies (Fig. 1). To eliminate the magnetic-field dependence of the spectrometer energy from our data, we evaluate $E_{L}=-e \alpha V_{L}+E_{S}$ according to Eq. (2) and plot $d E_{L} / d\left(\hbar \omega_{c}\right)$ in Fig. 4(d). For $M$ occupied Landau bands, Eq. (3) predicts that $d E_{L} / d\left(\hbar \omega_{c}\right)$ is in the range of $1 / 2 \leq d E_{L} / d\left(\hbar \omega_{c}\right) \leq M / 2$ (using $n=M-1$ ). The limits are set by the lowest and highest occupied Landau band. Our experimental data for occupied states close to $\mu_{L}$ lie exactly in this range for $M \geq 2$. In the magnetic quantum limit of $M=1$, the measured values are slightly higher than $1 / 2$ (experimental error bars are estimated to $1 / 4$, mainly due to uncertainties in $\alpha$ and $E_{S}$ ).

Finally, we discuss the role of inelastic processes in our experiment. The LDOS resonances of occupied states persist in Fig. 3 up to highest bias, whereas the resonances of empty states vanish below $V \approx-35 \mathrm{mV}$ (marked by a dotted line). To understand this phenomenon, we study the relaxation of tunneled electrons in the left contact for $V<0$ [Fig. 1(b)]. Their excess energy $\Delta E$ above $\mu_{L}$ lies in the range of $e\left(|V|+\left|V_{S}\right|\right) / 2 \leq \Delta E \leq e|V|$. If $\Delta E$ reaches the order of the Fermi energy, inelastic electron-electron scattering becomes as fast as elastic electron-impurity scattering. Thus, the interference of diffusive electron waves is destroyed and the LDOS fluctuations vanish. With a Fermi energy of $30 \mathrm{meV}$ and $\left|V_{S}\right| \sim 10 \mathrm{mV}$, we expect the LDOS resonances of empty states to disappear in the range of $30 \leq|V| \leq 50 \mathrm{mV}$, which is in agreement with experiment. At similar bias, also the emission of plasmon-optical-phonon coupled modes acts as an efficient source for dephasing (when $\Delta E$ exceeds $\hbar \omega_{p} \approx 26 \mathrm{meV}$ and $\hbar \omega_{L O} \approx 36 \mathrm{meV}$ ) [17]. The energy deficit (with respect to $\mu_{L}$ ) of the "holes" generated by tunneling in the left Fermi sea for $V<0$ lies in the range of $0 \leq \Delta E \leq e\left(|V|-\left|V_{S}\right|\right) / 2$ [Fig. 1(a)], being far smaller than the excess energy of tunneled electrons in the collector. Hence, the hole relaxation is much slower and the LDOS resonances from occupied states persist to highest bias voltages. Different dephasing rates explain also why the resonances from occupied states are stronger than those from empty states; cf. Fig. 3.

In summary, we studied resonant tunneling through impurity states in an asymmetric double-barrier het- erostructure. Depending on the direction of current flow, tunneling electrons probe the local density of either occupied or empty states (LDOS) in the heavily doped GaAs contact adjacent to the thicker barrier. At strong magnetic fields, the LDOS exhibits fan-type resonances in the energy-magnetic-field plane, which reflect the interplay of Landau quantization and quantum interference of scattered electron waves in the disordered bulk conductor. High above the Fermi level, the LDOS resonances disappear as a consequence of inelastic broadening.

We acknowledge support from the Bundesministerium für Bildung, Wissenschaft, Forschung und Technologie and from EPSRC (V.I. F.).

[1] For reviews, see Mesoscopic Phenomena in Solids, edited by B.L. Altshuler, P. A. Lee, and R. A. Webb (NorthHolland, Amsterdam, 1991).

[2] A. A. Abrikosov and I. A. Ryzhkin, Adv. Phys. 27, 147 (1978).

[3] D. G. Polyakov, Zh. Eksp. Teor. Fiz. 84, 749 (1983) [Sov. Phys. JETP 57, 433 (1983)]; 90, 546 (1986) [63, 317 (1986)]; in 20th International Conference on the Physics of Semiconductors, edited by J. Joannopoulos (World Scientific, Singapore, 1991), p. 2321.

[4] I. V. Lerner, Phys. Lett. A 133, 253 (1988).

[5] I. V. Lerner and M.E. Raikh, Phys. Rev. B 45, 14036 (1992); A. Y. Zyuzin and B.Z. Spivak, Zh. Eksp. Teor. Fiz. 98, 1011 (1990) [Sov. Phys. JETP 71, 563 (1990)].

[6] M.F. Crommie, C.P. Lutz, and D. M. Eigler, Nature (London) 363, 524 (1993); Y. Hasegawa and Ph. Avouris, Phys. Rev. Lett. 71, 1071 (1993).

[7] T. Schmidt et al., Europhys. Lett. 36, 61 (1996).

[8] M. W. Dellow et al., Phys. Rev. Lett. 68, 1754 (1992).

[9] A. K. Geim et al., Phys. Rev. Lett. 72, 2061 (1994).

[10] M. R. Deshpande et al., Phys. Rev. Lett. 76, 1328 (1996).

[11] M. R. Deshpande et al., in 22nd International Conference on the Physics of Semiconductors, edited by D. J. Lockwood (World Scientific, Singapore, 1995), p. 1899; P. J. McDonnell et al., Physica (Amsterdam) 211B, 433 (1995); Solid State Electron. 40, 409 (1996); T. Schmidt et al., ibid. 40, 15 (1996); J. W. Sleight et al., Phys. Rev. B 53, 15727 (1996).

[12] L. I. Glazman and K. A. Matveev, Pis'ma Zh. Eksp. Teor. Fiz. 48, 403 (1988) [JETP Lett. 48, 445 (1988)].

[13] L. Y. Chen and C. S. Ting, Phys. Rev. B 44, 5916 (1991).

[14] The bias-polarity independence of $\alpha$ indicates that $S$ is located closer to the left barrier than to the right one in the quantum well [for details, see T. Schmidt et al., Phys. Rev. B 55, 2230 (1997)].

[15] For $B \perp I$, tunneling is suppressed at high fields. Nevertheless, fan-type resonances are observed in the regime of $4 \mathrm{~T}<B<10 \mathrm{~T}$. They are less pronounced than for $B \| I$, which may be either related to skipping-orbitrelated interface effects [B. R. Snell et al., Phys. Rev. Lett. 59, 2806 (1987)] or the overall suppression of tunneling.

[16] T. Schmidt et al., Phys. Rev. B 51, 5570 (1995).

[17] M. Giltrow et al., Phys. Rev. Lett. 75, 1827 (1995). 